\title{
Effect of voids on the propagation of waves in an elastic layer
}

\author{
PRANABES KANTI PAL ${ }^{1}$, D ACHARYA ${ }^{2}$ and P R SENGUPTA ${ }^{3}$ \\ ${ }^{1}$ Indian Institute of Mechanics of Continua, 201, Manicktola Main Road, Flat \\ No 42, Calcutta 700 054, India \\ ${ }^{2}$ Department of Mathematics, Mahadevananda College, Barrackpore 743 101, \\ India \\ ${ }^{3}$ Department of Mathematics, University of Kalyani, Kalyani 741235 , India
}

MS received 26 September 1995

\begin{abstract}
The present paper investigates the propagation of waves in an elastic layer containing voids. Numerical calculations and discussions indicate that the velocity of the propagation of waves decreases due to the presence of voids in the material medium of the layer and the voids cause dispersion of the general waveform.
\end{abstract}

Keywords. Propagation of waves; distribution of voids; surface stress; volume fraction field; wave velocity equation; surface waves.

\section{Introduction}

Recently, the theory of elasticity concerning the solid elastic material consisting of a distribution of various pores, generally known as voids or vacuous pores, is receiving greater attention due to its theoretical and practical relevance. The general theory in this respect has been formulated by Nunziato and Cowin (Nunziato \& Cowin 1979; Cowin \& Nunziato 1983). They also formulated the linearised version of the above theory (Cowin \& Nunziato 1983) where the voids have been included as an additional kinematic variable. This theory reduces to the classical theory of elasticity in the limiting case when the void-volume vanishes. This new theory can play an important role in practical problems of geological and synthetic porous media where the classical theory is inadequate. Some basic theorems and a brief account of the theory on voids have been introduced by lesan (1985) and Cowin (1984) respectively. Cowin (1984) presented the inter-relationship between this theory of voids and other theories of elasticity. The uniqueness theorem in the theory of elastic material with voids has been presented by Chandrasekharaiah (1987b). He investigated plane waves in a rotating elastic solid with voids (Chandrasekharaiah 1987c). The effect of surface stresses and voids on Rayleigh waves in an elastic medium was also investigated by him (Chandrasekharaiah 1987c). Following the above theory, an attempi has been made in this paper to carry out a thorough investigation of the propagation of waves and vibrations 
in an isotropic, homogeneous, elastic solid layer containing a distribution of voids. The authors believe that the problem in its present form has not been discussed so far. In the present investigation, the results obtained are in agreement with the corresponding classical results when the parameter for the void character of the material medium tends to be zero.

\section{Formulation of the problem and boundary conditions}

Let us introduce a rectangular Cartesian frame of reference $O x_{1} x_{2} x_{3}$ in the middle plane of the elastic layer. We consider the effect of voids on the propagation of waves in an elastic layer of thickness $2 h$. The planes bounding the layer $x_{3}= \pm h$ are supposed to be free of stresses. There exist plane waves moving with a constant velocity $c$ in the positive direction of the $x_{1}$ axis. Both the longitudinal and transverse waves in the infinitely extended layer would be propagated. It is evident that the boundary surfaces of the elastic space lead to a distortion of the state of stress which also influences the velocity of propagation of elastic waves. Considering the nature of the problem we may take $u_{1}$ and $u_{3}$ as the non-zero components of the displacement $u$ at any point and they may be expressed in the form

$$
\begin{aligned}
& u_{1}=\frac{\partial P}{\partial x_{1}}-\frac{\partial Q}{\partial x_{3}}, \\
& u_{3}=\frac{\partial P}{\partial x_{3}}+\frac{\partial Q}{\partial x_{1}},
\end{aligned}
$$

where $P$ and $Q$ are displacement potentials which are functions of coordinates $x_{1}, x_{3}$ and time $t$. The dynamical equations of motion (Nunziato \& Cowin 1979; Cowin \& Nunziato 1983; Chandrasekharaiah 1987a) are

$$
\begin{aligned}
& \mu \nabla^{2} \mathbf{u}+(\mu+\lambda) \nabla \nabla \cdot \mathbf{u}+\beta \nabla \Phi=\partial^{2} u / \partial t^{2}, \\
& \alpha \nabla^{2} \Phi-\xi \Phi-\omega \frac{\partial \Phi}{\partial t}-\beta \nabla \cdot \mathbf{u}=\rho k \partial^{2} \varphi / \partial t^{2} .
\end{aligned}
$$

$\varphi$ is volume-fraction field; $\lambda, \mu$ are Lamé elastic constants, $\rho$ is the mass density; $\alpha, \beta, \xi, \omega$ and $k$ are new material constants characterizing the presence of voids.

For a plane deformation parallel to the $x_{1} x_{3}$ plane we take

$$
\mathbf{u}=\left(u_{1}, 0, u_{3}\right) .
$$

From (1), (2) and (4), we get the following differential equations

$$
\begin{aligned}
& \left(\nabla^{2}-\frac{1}{a^{2}} \frac{\partial^{2}}{\partial t^{2}}\right) P=-\frac{\beta}{\lambda+2 \mu} \Phi, \\
& \left(\nabla^{2}-\frac{1}{b^{2}} \frac{\partial^{2}}{\partial t^{2}}\right) Q=0 .
\end{aligned}
$$

Eliminating $\Phi$ from (3) and (5) we obtain

$$
\left[\left(\nabla^{2}-\frac{1}{a^{2}} \frac{\partial^{2}}{\partial t^{2}}\right)\left\{\nabla^{2}-\frac{1}{\alpha^{*}}\left(1+\omega^{*} \frac{\partial}{\partial t}+k^{*} \frac{\partial^{2}}{\partial t^{2}}\right)\right\}+\beta^{*} \nabla^{2}\right] P=0,
$$


where

$$
\begin{aligned}
& a^{2}=\frac{\lambda+2 \mu}{\rho}, \quad b^{2}=\frac{\mu}{\rho}, \quad \alpha^{*}=\frac{\alpha}{\xi}, \quad \omega^{*}=\frac{\omega}{\xi}, \\
& K^{*}=\frac{\rho K}{\xi}, \quad \beta^{*}=\frac{\beta^{2}}{\alpha(\lambda+2 \mu)} .
\end{aligned}
$$

In presence of voids the stress tensor $\sigma_{i j}$ obeys the following law (Nunziato \& Cowin 1979)

$$
\sigma_{i j}=\lambda \delta_{i j} u_{k, k}+\mu\left(u_{i, j}+u_{j, i}\right)+\beta \delta_{i j} \Phi \text {. }
$$

We seek solutions of (5) and (6) subject to the boundary conditions

$$
\sigma_{33}=\sigma_{31}=0, \text { on planes } x_{3}= \pm h \text {. }
$$

Following Nunziato \& Cowin (1979) we take the boundary condition due to the void nature of the material medium as

$$
\partial \Phi / \partial x_{3}=0, \text { for } x_{3}= \pm h .
$$

Now using (1), (4), (5) in (9) we get

$$
\begin{aligned}
& \sigma_{31}=\mu\left[2 \frac{\partial^{2} P}{\partial x_{1} \partial x_{3}}-\frac{\partial^{2} Q}{\partial x_{3}^{2}}+\frac{\partial^{2} Q}{\partial x_{1}^{2}}\right] \\
& \sigma_{33}=2 \mu\left[2 \frac{\partial^{2} Q}{\partial x_{1} \partial x_{3}}-\frac{\partial^{2} P}{\partial x_{1}^{2}}\right]+\frac{\mu}{b^{2}} \frac{\partial^{2} P}{\partial t^{2}}
\end{aligned}
$$

\section{Method of solution}

To solve (6) and (7) we take $P$ and $Q$ in the following forms

$$
[P, Q]=\left[\bar{P}\left(x_{3}\right), \bar{Q}\left(x_{3}\right)\right] \exp i\left(\eta x_{1}-\zeta t\right),
$$

where $\zeta$ is the angular frequency which is a real constant in our problem and $\bar{P}\left(x_{3}\right), \bar{Q}\left(x_{3}\right)$ are functions of $x_{3}, \eta$ is an unknown complex constant and $i=\sqrt{-1}$.

Introducing (12) in (6) and (7) we get the following differential equations

$$
\begin{aligned}
& {\left[\{ \frac { \mathrm { d } ^ { 2 } } { \mathrm { d } x _ { 3 } ^ { 2 } } - \eta ^ { 2 } ( 1 - \frac { \zeta ^ { 2 } } { a ^ { 2 } \eta ^ { 2 } } ) \} \left\{\frac{\mathrm{d}^{2}}{\mathrm{~d} x_{3}^{2}}-\eta^{2}-\frac{1}{\alpha^{*}}\left(1-i \zeta w^{*}-\zeta^{2} K^{*}\right)\right.\right.} \\
& \left.\left.+\beta^{*}\left(\frac{\mathrm{d}^{2}}{\mathrm{~d} x_{3}^{2}}-\eta^{2}\right)\right\}\right] P=0 \\
& \frac{\mathrm{d}^{2} Q}{\mathrm{~d} x_{3}^{2}}-\left(\eta^{2}-\frac{\zeta^{2}}{b^{2}}\right) Q=0
\end{aligned}
$$


Solutions of (13) and (14) are taken in the following form

$$
\begin{aligned}
& \bar{P}=A_{1} \sinh \eta m_{1} x_{3}+A_{2} \cosh \eta m_{1} x_{3}+A_{3} \sinh \eta m_{2} x_{3}+A_{4} \cosh \eta m_{2} x_{3}, \\
& \bar{Q}=A_{5} \sinh \eta m_{3} x_{3}+A_{6} \cosh \eta m_{3} x_{3},
\end{aligned}
$$

where $m_{1}$ and $m_{2}$ are the roots with positive real parts of the equation

$$
\begin{gathered}
\left(1-m^{2}\right)^{2}-\left[r^{2}-\frac{1}{\eta^{2} \alpha^{*}}\left(1-i \zeta \omega^{*}-\zeta^{2} K^{*}\right)+\frac{1}{\eta^{2}} \beta^{*}\right]\left(1-m^{2}\right) \\
-\frac{r^{2}}{\eta^{2} \alpha^{*}}\left(1-i \zeta \omega^{*}-\zeta^{2} K^{*}\right)=0
\end{gathered}
$$

and

$$
m_{3}=\left(1-s^{2}\right)^{\frac{1}{2}}
$$

$A_{1}, A_{2}, A_{3}, A_{4}, A_{5}, A_{6}$ are arbitrary constants and

$$
c=\frac{\zeta}{\eta}, r=\frac{c}{a}, s=\frac{c}{b}
$$

Applying the boundary conditions (10) and (10a) one obtains the following:

$$
\begin{aligned}
& 2 i m_{1} q_{1} A_{1}+2 i m_{1} p_{1} A_{2}+2 i m_{2} q_{2} A_{3}+2 i m_{2} p_{2} A_{4}-\left(1+m_{3}^{2}\right) p_{3} A_{5} \\
& \quad-\left(1+m_{3}^{2}\right) q_{3} A_{6}=0 \\
& 2 i m_{1} q_{1} A_{1}-2 i m_{1} p_{1} A_{2}+2 i m_{2} q_{2} A_{3}-2 i m_{2} p_{2} A_{4}+\left(1+m_{3}^{2}\right) p_{3} A_{5} \\
& \quad-\left(1+m_{3}^{2}\right) q_{3} A_{6}=0 \\
& \quad\left(2-s^{2}\right) p_{1} A_{1}+\left(2-s^{2}\right) q_{1} A_{2}+\left(2-s^{2}\right) p_{2} A_{3}+\left(2-s^{2}\right) q_{2} A_{4} \\
& \quad+2 i m_{3} q_{3} A_{5}+2 i m_{3} p_{3} A_{6}=0 \\
& \left(2-s^{2}\right) p_{1} A_{1}-\left(2-s^{2}\right) q_{1} A_{2}+\left(2-s^{2}\right) p_{2} A_{3}-\left(2-s^{2}\right) q_{2} A_{4} \\
& \quad-2 i m_{3} q_{3} A_{5}+2 i m_{3} p_{3} A_{6}=0 \\
& m_{1} n_{1} q_{1} A_{1}+m_{1} n_{1} p_{1} A_{2}+m_{2} n_{2} q_{2} A_{3}+m_{2} n_{2} p_{2} A_{4}=0 \\
& m_{1} n_{1} q_{1} A_{1}-m_{1} n_{1} p_{1} A_{2}+m_{2} n_{2} q_{2} A_{3}-m_{2} n_{2} p_{2} A_{4}=0
\end{aligned}
$$

where

$$
\begin{aligned}
p_{j} & =\sinh \eta m_{j} h, \\
q_{j} & =\cosh \eta m_{j} h, \quad j=1,2,3, \\
n_{1} & =m_{1}^{2}+r^{2}-1, . \\
n_{2} & =m_{2}^{2}+r^{2}-1 .
\end{aligned}
$$

Elimination of the constants in (18) gives

$$
\Delta=\operatorname{det}\left[a_{i j}\right]=0, \quad i, j=1,2,3,4,5,6,
$$

where

$$
a_{11}=2 i m_{1} q_{1}, a_{12}=2 i m_{1} p_{1}, a_{13}=2 i m_{2} q_{2}, a_{14}=2 i m_{2} p_{2},
$$




$$
\begin{aligned}
& a_{15}=-\left(1+m_{3}^{2}\right) p_{3}, a_{16}=-\left(1+m_{3}^{2}\right) q_{3}, \\
& a_{21}=2 i m_{1} q_{1}, a_{22}=-2 i m_{1} p_{1}, a_{23}=2 i m_{2} q_{2}, a_{24}=-2 i m_{2} p_{2}, \\
& a_{25}=\left(1+m_{3}^{2}\right) p_{3}, a_{26}=-\left(1+m_{3}^{2}\right) q_{3}, \\
& a_{31}=\left(2-s^{2}\right) p_{1}, q_{32}=\left(2-s^{2}\right) q_{1}, a_{33}=\left(2-s^{2}\right) p_{2}, \\
& a_{34}=\left(2-s^{2}\right) q_{2}, a_{35}=2 i m_{3} q_{3}, a_{36}=2 i m_{3} p_{3}, \\
& a_{41}=\left(2-s^{2}\right) p_{1}, a_{42}=-\left(2-s^{2}\right) q_{1}, a_{43}=\left(2-s^{2}\right) p_{2}, \\
& a_{44}=-\left(2-s^{2}\right) q_{2}, a_{45}=-2 i m_{3} q_{3}, a_{46}=2 i m_{3} p_{3}, \\
& a_{51}=m_{1} n_{1} q_{1}, a_{52}=m_{1} n_{1} p_{1}, a_{53}=m_{2} n_{2} q_{2}, a_{54}=m_{2} n_{2} p_{2}, \\
& a_{55}=0, a_{56}=0, \\
& a_{61}=m_{1} n_{1} q_{1}, a_{62}=-m_{1} n_{1} p_{1}, a_{63}=m_{2} n_{2} q_{2}, a_{64}=-m_{2} n_{2} p_{2}, \\
& a_{65}=0, a_{66}=0
\end{aligned}
$$

Equation (20) represents the wave velocity equation for surface waves in an elastic layer with voids. This equation contains $c$ and $\eta$ as only unknown quantities and hence $c$ can be expressed as a function of $\eta$ indicating the dispersive nature of waves considered. This dispersive nature of the general waveform arises due to the presence of voids in the material medium. The above sixth-order determinant $\Delta$ can be expressed as the product of two third-order determinants as follows:

$$
\Delta=\Delta_{1} \cdot \Delta_{2}
$$

where

$$
\Delta_{1}=\left|\begin{array}{ccc}
\frac{m_{1} n_{1}}{m_{2} n_{2}} \tanh \eta m_{1} h & \tanh \eta m_{2} h & 0 \\
2 m_{1} \tanh \eta m_{1} h & 2 m_{2} \tanh \eta m_{2} h & \left(1+m_{3}^{2}\right) \tanh \eta m_{3} h \\
2-s^{2} & 2-s^{2} & 2 m_{3}
\end{array}\right|
$$

and

$$
\Delta_{2}=\left|\begin{array}{ccc}
\frac{m_{1} n_{1}}{m_{2} n_{2}} & 1 & 0 \\
2 m_{1} & 2 m_{2} & 1+m_{3}^{2} \\
\left(2-s^{2}\right) \tanh \eta m_{1} h & \left(2-s^{2}\right) \tanh \eta m_{2} h & 2 m_{3} \tanh \eta m_{3} h
\end{array}\right| .
$$

Hence (20) implies either $\Delta_{1}=0$ or $\Delta_{2}=0$.

We now discuss each of the above cases separately as follows

Case A $\left(\Delta_{1}=0\right)$ : After simplification, $\Delta_{1}=0$ gives

$$
\Delta_{1}^{\prime}=\frac{m_{1} n_{1}}{m_{2} n_{2}} \frac{\tanh \eta m_{1} h}{\tanh \eta m_{2} h} \cdot \Delta_{1}^{\prime \prime},
$$

where

$$
\Delta_{1}^{\prime}=\left|\begin{array}{cc}
2 m_{1} \tanh \eta m_{1} h & \left(1+m_{3}^{2}\right) \tanh \eta m_{3} h \\
2-s^{2} & 2 m_{3}
\end{array}\right|
$$




$$
\Delta_{1}^{\prime \prime}=\left|\begin{array}{cc}
2 m_{2} \tanh \eta m_{2} h & \left(1+m_{3}^{2}\right) \tanh \eta m_{3} h \\
2-s^{2} & 2 m_{3}
\end{array}\right|
$$

Case A1: If the length of the wave is large in comparison with the thickness of the layer $2 h$, the hyperbolic tangents can be replaced by their arguments. So (21) becomes

$$
4 R^{2} V^{* 2}-\left(2-s^{2}\right)^{2}=0,
$$

where

$$
\begin{aligned}
& R^{2}=1-r^{2}, R_{1}^{2}=\left(m_{1} / R\right)^{2}, \\
& R_{2}^{2}=\left(m_{2} / R\right)^{2}, V^{* 2}=R_{1}^{2} R_{2}^{2} /\left(R_{1}^{2}+R_{2}^{2}-1\right) .
\end{aligned}
$$

Equation (22) determines the wave velocity of plane waves and corresponds to results similar to those obtained by Rayleigh (1889) and Lamb (1916) in an elastic layer containing some voids. When the medium is free from voids we have $m_{1}=R, R_{1}=1, V^{*}=1$ and we get the classical results of Rayleigh (1889) and Lamb (1916). Thus we note that wave velocity due to Rayleigh and Lamb in presence of voids may be obtained from the corresponding classical form by replacing $R$ by $R V^{*}$ where $V^{*}$ is given by (23). For small frequency waves we ignore higher degree terms in $\zeta$ (Chandrasekharaiah 1987a). In view of this approximation and with the help of (8), (15) and (17), (22) becomes

$$
4 V_{0}^{2}-\left(2-s^{2}\right)^{2}=0
$$

where

$$
V_{0}=\left[1-\left(c^{2} / a^{2}(1-N)\right)\right]^{\frac{1}{2}}
$$

and

$$
N=\alpha^{*} \beta^{*}=\beta^{2} /[\xi(\lambda+2 \mu)] .
$$

Case A2: If the length of the wave is very small in comparison with the thickness of the layer $2 h$, we may assume that the ratio of hyperbolic tangents in (21) approaches unity and hence (21) becomes

$$
4 m_{3} R R^{*}-\left(2-s^{2}\right)^{2}=0,
$$

where

$$
\begin{aligned}
R & =\left(1-r^{2}\right)^{\frac{1}{2}}, R_{1}=m_{1} / R, \\
R_{2} & =m_{2} / R, R^{*}=R_{1} R_{2}\left(R_{1}+R_{2}\right) /\left[R_{1}^{2}+R_{2}^{2}+R_{1} R_{2}-1\right] .
\end{aligned}
$$

Equation (27) determines the velocity of Rayleigh surface waves in an elastic layer with voids. For small frequency waves, which play a great role in analysing motions caused by earthquakes and explosions, we neglect the higher degree terms in $\zeta$ (Chandrasekharaiah 1987a). With the use of this approximation equation (27) transforms to

$$
4 m_{3} R_{0}-\left(2-s^{2}\right)^{2}=0,
$$

where

$$
R_{0}=\left[1-\left(c^{2} / a^{2}(1-N)\right)\right]^{\frac{1}{2}}, \quad N=\beta^{2} /[\xi(\lambda+2 \mu)]
$$


Case $B\left(\Delta_{2}=0\right)$ : On simplification, $\Delta_{2}=0$ gives

$$
\begin{aligned}
& 4 m_{1} m_{3}-\left(1+m_{3}^{2}\right)\left(2-s^{2}\right) \frac{\tanh \eta m_{1} h}{\tanh \eta m_{3} h} \\
& =\frac{m_{1} n_{1}}{m_{2} n_{2}}\left[4 m_{2} m_{3}-\left(1+m_{3}^{2}\right)\left(2-s^{2}\right) \frac{\tanh \eta m_{2} h}{\tanh \eta m_{3} h}\right] .
\end{aligned}
$$

Case B1: If the length of the wave is large in comparison with thickness of the layer, the hyperbolic tangents can be replaced by the first two terms of their expansions into series and hence (31) becomes

$$
\begin{gathered}
4 m_{1} m_{3}-\left(1+m_{3}^{2}\right)\left(2-s^{2}\right)\left[m_{1}\left(1-\frac{1}{3} \eta^{2} h^{2} m_{1}^{2}\right) / m_{3}\left(1-\frac{1}{3} \eta^{2} h^{2} m_{3}^{2}\right)\right] \\
=\left(m_{1} n_{1} / m_{2} n_{2}\right)\left\{4 m_{2} m_{3}-\left(1+m_{3}^{2}\right)\left(2-s^{2}\right)\right. \\
\left.\quad \times\left[m_{2}\left(1-\frac{1}{3} \eta^{2} h^{2} m_{2}^{2}\right) / m_{3}\left(1-\frac{1}{3} \eta^{2} h^{2} m_{3}^{2}\right)\right]\right\} .
\end{gathered}
$$

Equation (32) may be regarded as the revised form of the classical result obtained by Rayleigh (1889) and Lamb (1916) in an elastic layer with voids. If the layer is free from voids $(\Phi=0),(32)$ simplifies to the form

$$
c^{2} / b^{2}=(4 / 3) \eta^{2} h^{2}\left(1-\left(b^{2} / a^{2}\right)\right)
$$

which is the classical result of Rayleigh (1889) and Lamb (1916).

Case B2: If the length of the wave is small in comparison with the thickness of the layer, the ratio of hyperbolic tangents in (31) may be approximated to unity and (31) reduces to (27) which determines the velocity of Rayleigh surface waves in an elastic layer with voids.

\section{Numerical results}

From (24) we obtain

$$
s=2\left\{1-1 /\left[\left(a^{2} / b^{2}\right)(1-N)\right]\right\}^{\frac{1}{2}} .
$$

Table 1. Values of $s$ for case A1.

\begin{tabular}{llllllllll}
\hline \multicolumn{10}{c}{$(a / b)^{2}$} \\
\hline $\mathrm{N}$ & 2.3710 & 2.4758 & 2.5806 & 2.6854 & 2.7902 & 2.8950 & 2.9980 & 3.5 & 4.3 \\
\hline 0 & 1.5208 & 1.5441 & 1.5652 & 1.5844 & 1.6020 & 1.6181 & 1.6327 & 1.6903 & 1.7521 \\
0.2000 & 1.3752 & 1.4073 & 1.4361 & 1.4622 & 1.4859 & 1.5076 & 1.5272 & 1.6036 & 1.6844 \\
0.3000 & 1.2609 & 1.3008 & 1.3363 & 1.3682 & 1.3972 & 1.4234 & 1.4471 & 1.5386 & 1.6343 \\
0.4000 & 1.0901 & 1.1434 & 1.1902 & 1.2318 & 1.2691 & 1.3028 & 1.3328 & 1.4475 & 1.5651 \\
0.5000 & 0.7911 & 0.8768 & 0.9487 & 1.0104 & 1.0643 & 1.1120 & 1.1539 & 1.3093 & 1.4627 \\
\hline
\end{tabular}


Table 2. Values of $s$ for case A2.

\begin{tabular}{lllllllllr}
\hline \multicolumn{10}{c}{$(a / b)^{2}$} \\
\hline $\mathrm{N}$ & 2.3710 & 2.4758 & 2.5806 & 2.6854 & 2.7902 & 2.8950 & 2.9980 & 3.5 & 4.3 \\
\hline 0 & 0.8996 & 0.9042 & 0.9082 & 0.9116 & 0.9145 & 0.9171 & 0.9194 & 0.9274 & 0.9331 \\
0.2000 & 0.8627 & 0.8721 & 0.8799 & 0.8865 & 0.8920 & 0.8968 & 0.9009 & 0.9142 & 0.9254 \\
0.3000 & 0.8221 & 0.8375 & 0.8501 & 0.8605 & 0.8692 & 0.8766 & 0.8827 & 0.9030 & 0.9188 \\
0.4000 & 0.7406 & 0.7686 & 0.7913 & 0.8100 & 0.8254 & 0.8383 & 0.8489 & 0.8797 & 0.9079 \\
0.5000 & 0.5547 & 0.6115 & 0.6574 & 0.6951 & 0.7263 & 0.7524 & 0.7739 & 0.8406 & 0.8830 \\
\hline
\end{tabular}

Values of $s$ for different values of $(a / b)^{2}$ and $N$ for case $\mathrm{A} 1$ are shown in table 1.

It is observed from table 1 that the wave velocity decreases with the increase of values of $N$ for a particular value of $(a / b)^{2}$. We further note that for a particular value of $N$, the wave velocity increases with the increase of $(a / b)^{2}$.

Again, from (29) one obtains

$$
s^{6}-8 s^{4}+\left\{24-16 /\left[\left(a^{2} / b^{2}\right)(1-N)\right]\right\} s^{2}-\left\{16-16 /\left[\left(a^{2} / b^{2}\right)(1-N)\right]\right\}=0 .
$$

Values of $s$ for different values of $N$ and $(a / b)^{2}$ for case A2 are shown in table 2.

From (22), (27) and (32) we see that the wave velocity equation contains $c$ and $\eta$ as the only unknown quantities and hence $c$ can be expressed as a function of $\eta$ in each case indicating the dispersive nature of the waves.

Table 2 reveals that the Rayleigh wave velocity in the presence of voids in an elastic layer decreases when the value of $N$ increases for a particular value of $(a / b)^{2}$. Also for a particular value of $N$, the Rayleigh wave velocity increases with the increase of values of $(a / b)^{2}$.

Similar computations may be made and conclusions drawn for the cases in $B$.

\section{Conclusions}

The most significant outcome of the paper is that voids modulate the surface waves by reducing their speed as well as by causing dispersion.

The authors are very grateful to the reviewer for his/her valuable comments and suggestions towards the improvement of this paper.

\section{References}

Chandrasekharaiah D S 1987a Effects of surface stresses and voids on Rayleigh waves in an elastic solid. Int. J. Eng. Sci. 25: 205-211

Chandrasekharaiah D S 1987b A uniqueness theorem in the theory of elastic materials with voids. J. Elast. 18: 173-179

Chandrasekharaiah D S 1987c Plane waves in a rotating elastic solid with voids. Int. J. Eng. Sci. 25: 591-596 
Cowin S C 1984 The stresses around a hole in a linear elastic material with voids. Q. J. Mech. Appl. Math. 37: 441-465

Cowin S C, Nunziato J W 1983 Linear elastic materials with voids. J. Elast. 13: 125-147

Iesan D 1985 Some theorems in the theory of elastic materials with voids. J. Elast. 15: 215-224

Lamb H 1916 On waves in an elastic plate. Proc. $R$. Soc. 93: 114-128

Nunziato J W, Cowin S C 1979 A nonlinear theory of elastic materials with voids. Arch. Ration. Mech. Anal. 72: 175-201

Pal P K, Acharya D, SenGupta P R 1994 Surface waves in elastic solids with voids. Proc. Int. AMSE Conf. on Signals, Data and Systems (Hyderabad: AMSE Press) vol. 2, pp. 17-32

Rayleigh F W 1889 On the free vibrations of an infinite plate of homogeneous isotropic elastic material. Proc. Math. Soc. 20: 225-234

SenGupta P R 1967 On the problem of two layered semi-infinite body of transversely isotropic materials. Indian J. Mech. Math. 5

SenGupta P R, Ghosh B 1974 Effect of couple-stresses on propagation of waves in an elastic layer. Pure Appl. Geophys. 112: 331-338 